\title{
Hypothyroidism, CTCAE
}

National Cancer Institute

\section{Source}

National Cancer Institute. Hypothyroidism, CT CAE. NCI Thesaurus. Code C143576.

A disorder characterized by a decrease in production of thyroid hormone by the thyroid gland. 